\title{
Hirsutism-A Comprehensive Update of Embryology, Aetiopathogenesis and Therapeutic Approach
}

\author{
Kulvinder Kochar Kaur ${ }^{1 *}$, Gautam Allahbadia ${ }^{2}$ and Mandeep Singh ${ }^{3}$ \\ ${ }^{1}$ Scientific Director, Dr Kulvinder Kaur Centre for Human Reproduction, India \\ ${ }^{2}$ Scientific Director, Rotunda-A Centre for Human reproduction, India \\ ${ }^{3}$ Consultant Neurologist, Swami Satyanand Hospital, India
}

Submission: January 06, 2017; Published: February 03, 2017

*Corresponding author: Kulvinder kaur, Scientific Director, Dr Kulvinder Kaur Centre for Human Reproduction, 721,GT. Nagar, jalandhar-144001, Punjab, India, Tel: 91-181-9501358180; Fax: 91-181-4613422; Email: kulvinder.dr@gmail.com

\begin{abstract}
Hirsutism, defined as terminal hair on the body of a female, affects $5-10 \%$ of women. Of these the commonest cause is anovulation secondary to PCOS, which is a fairly common disorder affecting 6-10\% of women of reproductive age group. Although ovary is the main source of androgen excess, evidence with synthesis of androgens from adrenals is exaggerated with $25-50 \%$ of PCOS patients showing an adrenal androgen excess. Besides PCOS other hormonal causes like thyroid hormone deficiency,growth hormone deficiency, or hyperprolactinemia, hyperinsulinemia which may act on their own or synergistic increase with adrenal androgens. A serum T over $2 \mathrm{ng} / \mathrm{ml}$, warrants exploring for an androgen secreting tumor. Although in some cases of PCOS massive virilization deepening of voice, receding hairline with a normal karyotype has been reported in the absence of a tumor, it just needs simple treatment efforts of controlling hyperandrogenemia.

In this review we have comprehensively reviewed the embryology of hair growth, the aeitiopathogenesis of hirsutism along with therapeutic approach. Contrary to commonly used preparations containing cyproterone, oral contraceptives like preparations having low dose ethinyl estradiol and drosperinone with low dose flutamide $75 \mathrm{mg}$ remains the choice of treatment in unmarried girls not desiring pregnancy and in married women also it is these OC's for 6 months may be the first choice of drug and although ovulation induction and insulin sensitizers may help in achieving pregnancy they do not treat the hirsutism. Role of spironolactone cream, finasteride cream are discussed as well. Although eflornithine hydrochloride (Vasqua) is available as a facial cream, the author has not found its efficacy and hence it is not discussed. Similarly although other agents like cemetidine may have some effects, they are not the primary drugs of choice, hence are not discussed.
\end{abstract}

Abbreviations: PCOS: Polycystic Ovary Syndrome; NCAH: Non Classical Adrenal Hyperplasia; IH: Idiopathic Hirsutism; FU: Follicular Unit; SHBG: Sex Hormone Binding Globulin; CT: Computed Tomography; MRI: Magnetic Resonance Imaging; USG: Ultrasonography

\section{Introduction}

Hirsutism is defined as the terminal hair on the body of a female patient which is distributed in a male like pattern.It affects roughly $5-10 \%$ of women [1-3]. Presence of hirsutism evokes lot of stress and affects psychosocial development [46]. In most women hirsutism indicates, the presence of one of the conditions like the polycystic ovary syndrome (PCOS), androgen producing tumors, non classical adrenal hyperplasia (NCAH)orsyndromes of severe insulin resistance,than being an isolated disorder. Occasionally it may not be because of any of these causes when it is termed as idiopathic hirsutism (IH) or peripheral hirsutism. Although often it is referred to as familial hirsutism, but this should not be used for IH as it has been shown

that other causes of hyperandrogenism like PCOS and NCAH, show a strong familial distribution $[7,8]$.

\section{Embryology of hair growth}

Hair follicle development starts at around 9-12 weeks $[9,10]$, and gets derived from the epidermis.It is mainly a solid column of cells which proliferate starting from the basal layers of the epidermis and protrude downwards into the dermis. Once this elongates it meets a bundle of mesodermal cells (dermal papilla), which it envelops at the bulbous tip known as bulb. This solid epithelial column then hollows out to form a hair canal, and the pilosebaceous unit (a hair follicle, sebaceous glands and anterior 
pili muscles) get laid down. Colour of hair is decided by pigments which get produced by melanocytes in the bulb.

The growth of hair starts with the proliferation of epithelial cells at the base of the canal in contact with the dermal papilla. The fetus is covered with lanugo hair, which is lightly pigmented, has a thin diameter, short in length, and are attached very fragilely. Total endowment of hair follicles is made as early as 22 weeks of gestation age and no new follicles get produced denovo. The total number of hair are approximately 50million hair follicles, which cover the body,of these 100,000-150,000are present on the scalp, while rest are present on facial and body areas.Only areas which are devoid of hair are feet, palms of hand and lips $[11,12]$.

The concentration of hair follicles which get laid down / unit area of facial, skin does not differ materially between sexes but does differ between races and ethnic groupsof white>asia; mediteranean>Nordic.Also hair growth differences between different races reflects hair follicle differences in $5 \alpha$ - reductaseRA(5- $\alpha-R A)[13]$. This pattern is genetically determined.

\section{Hair structure and function}

Hair grows cyclically, rather than in a continuous fashion, having alternating phases of activity and inactivity. There are 3 cycles i) Anagen-the growing phase ii) Catagen-rapid involution phase iii) Telogen-quiescent phase.

In telogen/resting phase there are short hairs which are loosely attached to the base of the bulb of the epithelial canal. At the initiation of growth in anagen, matrix cells present at the base begin to proliferate followed by descending into the dermis. This epithelial column enlarges 4-6 times from the resting phase. On completion of downward growth, rapid growth of the matrix cells continuously, pushes upwards to the skin surface. The contact with previous hair is broken, followed by shedding of the hair. The superficial matrix cells form a keratinized column. On finishing, catagen or rapid involution of the column occurs by shrinkage, along with shriveling of the bulb, terminating in the resting phase or telogen.

The length of hair is determined by the time the hair grows in anagen. Scalp hair remains in anagen for 2-5years with a relative short resting phase. In the rest of body like forearm, a short anagen and a long telogen leads to short hair having stable nongrowing length. Presence of continuous hair growth/ shedding periodically depends on the degree to which individual hair follicles are asynchronous from their neighbours. Scalp hair being asynchronous, always seem to be growing. The resting phase of some hairs-approximately $10-15 \%$ is not apparent. In case marked synchrony is achieved, leading to simultaneous telogen at the same time, simultaneous shedding culminates in a process known as telogen effluvium. Rarely women complain of rapid hair loss from scalp, this being a temporary phenomenonlasting usually 6-8 months is followed by resumption of hair growth, on reestablishment of hair synchrony. Causes of telogen effluvium include, pregnancy, fever and drugs.Still measuring thyroid stimulating hormone is important to rule out any thyroid disease.

There are three types of hair structurally. Soft hair covering skin of fetus is lanugo which gets shed in 1-4 months postpartum. Vellus hairs are nonpigmented usually, measuring $<2 \mathrm{~mm}$ in length and cover the areas of body which are apparently hairless. Histologically vellus hairs have a diameter, which does not exceed $10.03 \mathrm{~mm}$, smaller in diameter as compared to the investing root sheath [14]. Lastly terminal hair is longer, coarse in texture and are pigmented. These make up the eyebrows, hairlashes, axillary and pubic hair in both sexes, and most of the body and facial hair in men [15]. These hairs are usually medullated. Innermost area of terminal hair follicles is called medulla, and is considered to be a collapsed protein' although its exact composition is controversial. This medulla is missing in lanugo and vellus hairs. Hair follicles form groups in skin, which are called follicular unit (FU), each FU consists of 3-4 follicles along with sebaceous glands and connective tissue sheath $[16,17]$. No gender difference exists regarding $\mathrm{FU}$, and basic difference in men and women don't relate to number of FU's but to the type and quality of hair.

Hypertrichosis implies increased hair of the fetal-lanugo type in a generalized fashion, usually secondary to drugs or malignancy. Vellus hairs are the ones present in prepubertal girls, which are unpigmented. While coarse, pigmented hair, which grow on various parts of the body during the adult years are referred to as terminal hairs. Hirsutism means a transformation from vellus to terminal hair.

\section{Factors influencing hair growth}

Dermal papilla holds the key regarding hair growth will continue despite any injury, freezing, xrays. Even with severe injury once dermal papilla survives, hair follicle will regenerate, followed by regrowth of the hair. Damaging the dermal papilla like by laser or electrolysis properly only ensures complete hair removal.

Basically sex steroids, multiple local factors as well as systemic factors act directly or indirectly on the dermal papilla for regulating the hair growth. Also the same factor may act on the rest of the hair follicle, which includes the outer and inner root sheaths and the follicular stem cells of the bulge area [18] and these may be as important as the dermal papilla for regulating the growth of hair. Of the local and systemic factors different growth factors, and cytokines, have been shown to affect hair growth [18-22]. Suggestions that these factors act by increasing the synthesis of stromolysin a matrix matalloproteinse which acts on the dermal papilla toincrease the growth [23]. Hormones like thyroid hormone (TH) $[24,25]$ and GH [26], also can affect hair growth. Generally it is the deficiency of TH/GH, be it disease associated or drug induced, is usually associated with changes in the anagen-telogen ratio in scalp and body hair $[26,27]$. 
In case of $\mathrm{TH}$ treatment in hypothyroid patients, usually leads to regrowth of scalp hair within approximately 8weeks [24]. TH receptors have been proved to be present on the outer sheath cells of the hair follicles, with a positive effect of c-T3 on the cellular proliferation in cultured hair follicles [28]. Even in GH deficient men who are treated with substitution of GH, shows an increase in body hair which occurs without any observable free androgen index [26].

This suggests that GH can directly stimulate body hair which is independent of circulating androgens getting raised. Binding of GH has been shown in the layers of the lower one thirds of the follicle, the outer sheath of the upper two third of the follicle and the dermal papilla [27]. Notwithstanding a direct growth effect of GH on 'invitro' follicles growth or morphology has not been observed [29]. There is a possibility that the effect of $\mathrm{GH}$ is modulated through insulin like growth $\mathrm{f}$ actor 1(IGF1). Fibroblasts in human scrotal skin showed IGF1 but no increase in IGFII or insulin, causing an increase in 5- $\alpha$ RA, an enzyme necessary for the potentiation of the androgen effect [29]. Also in culture conditions, IGF1, but not IGFII or insulin caused stimulation of hair follicle growth [30].

Of the sex steroids androgens are the most essential in deciding the distribution of hairs as well as types of hairs over the human body. Androgens can exert action like hair follicles which are producing vellus types of hair to convert them to produce terminal hair. Under in vivo conditions, exogenous androgens affect the differentiation of hair follicles in androgen sensitive areas of the body like genitalia and facial beard areas in normal males or eunuchs and female to male transsexuals [31].

Besides stimulating terminal hair production, androgens also prolong the anagen phase of the body hairs, while simultaneously reducing the anagen phase of scalp hairs [32,33]. They also increase sebum secretion. Thus they increase body hair along with their oiliness.

\section{Control of GnRH secretion in PCOS}

Mahesh VB reviewed the control of steroidogenesis investigation work of his from 1956 to 2010 regarding hirsutism, virilization and PCOS, They found that in ovariectomized rats $P$ was a major enhancer of $E$ induced gonadotropin release ovulatoryGn Surge. Manifestation of P effects were manifested by the depletion of their occupied E receptors of the anterior pituitary, hypothalamic GnRH release and inhibition of enzymes which degrade GnRH.P promotes the synthesis of selective LH release and acted using GABA A receptor system. ASelective release of FSH was induced by the $5 \alpha$ reduced metabolite of $\mathrm{P}$ and P's ability to selectively induce FSH release depended on getting converted to $5 \alpha$ reduced metabolite. Since GnRH neurons do not have steroid receptors, this effect was brought about by the excitatory amino acid glutamate, which further requires stimulation from nitic oxide. The effect of $\mathrm{P}$ was mediated on the 361 base pair region of the FSH promoter gene [34]. Although their has been controversy regarding Gn RH neurons having estrogen $\beta$ receptors, which may have a role,besides kisspeptin neurons, which also possess $\mathrm{E}$ receptors, multiple pathways may be utilized, and this effect of these hormones using the steroid receptors present on glutamatergic neurons may be additional mechanism [reviewed in ref 35].

\section{Androgen production}

Normally in females testosterone (T) production rate is around $0.2-0.3 \mathrm{mg} /$ day. Peripheral conversion of androstendione (An) contributes to approximately $50 \%$ of $\mathrm{T}$ whereas $25 \%$ of circulating $\mathrm{T}$ comes from adrenal gland and ovary respectively, except at midcycle where ovarian contribution is just $10-15 \%$. Dehydroepiandrosterone sulphate (DHAS) comes exclusively from adrenal gland, while $90 \%$ of dehydroepiandrosterone (DHA) comes from adrenal.

Of the circulating $\mathrm{T} 80 \%$ is bound to a $\beta$ globulin also known as sex hormone binding globulin (SHBG). Approx imately in women $19 \%$ is loosely bound to albumin,and $1 \%$ remains unbound.In contrast DHA, DHASand An do not bind to protein significantly,with the routine RIA reflecting the available biological activity of these hormones. While routine assays in case of T measure bound and unbound concentratons.Androgens decrease SHBG production in liver. Thus in men binding capacity is lower than in women with $2-3 \%$ of $\mathrm{T}$ circulating in free active form. Insulin decrease SHBG, while estrogen and thyroid hormone increase it. Hence in hyperthyroidism, pregnancy and estrogen containing medicines the binding capacity is in creased. Excess androgens depress SHBG in hirsute women, besides by hyperinsulinemia if present. The Total $\mathrm{T}$ maybe within normal limits in a hirsute woman. Since the very presence of hirsutism and/or masculinization, indicates increased andogen there is no indication for testing free T.One can presume lower binding capacity and raised freeT.

Only $25 \%$ of circulating T comes from peripheral conversion in hirsute women and maximum is due to direct glandular secretion. Data indicated with increased androgen production that ovary is the major source of raised $\mathrm{T}$ and An in hirsute woman [36]. Commonest cause of hirsutism in women is persistent anovulation, associated with increased androgen production. Adrenal causes are very uncommon.

\section{DHT metabolism}

Though $\mathrm{T}$ is the main circulating androgen, dihydroT (DHT), is the major nuclear androgen in many sensitive tissues, which includes hair follicles and the Pilosebaceous unit in the skin. DHT gets metabolized to $3-\alpha$-androstanediol in the peripheral tissues and its glucuronide form $3-\alpha A G$ is utilized in the form of a markerof target tissue cellular activity [37-38]. 3 $\alpha$-AGcorrelates with the level of 5- $\alpha$ reductase activity [(5- $\alpha$ RA) (T and An)] in the skin. This makes 3 important laboratory measurements i) T-which is a measure of ovarian and adrenal activity ii) DHASbeing a measure of adrenal activity iii) 3- $\alpha \mathrm{AG}$, a measure of 
peripheral target tissue activity.Hirsutism, basically is not a disorder of hair, but rather it shows the raisedactivity of 5- $\alpha \mathrm{RA}$, which reflects greater DHT production. Activity of this enzyme reflects greater availability of precursori. e. circulating T, which is the primary factor or by some unknown local tissue mechanisms which are still unclear. Once other laboratory measurements are normal raised 3- $\alpha$ AG reflects an increased activity of 5- $\alpha$ RA in the peripheral component [39]. But 3-AGcan also reflect conjugation activity in liver and the effect of various major precursors derived from adrenal and not from peripheral sources [40] Thus 3- $\alpha \mathrm{AG}$ is not the sole measure of skin androgen metabolism. Usually there is not much benefit of testing 3- $\alpha \mathrm{AG}$ as i) it is not an absolute measurement with $20 \%$ overlap in hirsute women and ii) the ultimate final treatment is not going to affect either the diagnosis or the therapy.

\section{Prostate specific antigen (PSA)}

PSA is basically a serine protease produced by the prostate gland, thus used as a tumor marker for diagnosis along with management of prostate cancer. PSA has also been detected in female tissues, measurable by very sensitive assays. Since androgens increase PSA gene, one can expect increased circulating PSA in women with hyperandrogenism. Circulating levels of 3-a AG correlate with the increased PSA in hirsute women [41].There is no response to ovarian/adrenal stimulation, which indicates a widespread origin $[42,43]$. Right now no clinical applications of measuring PSA exist in women.

\section{Scoring degree of hirsutism}

On the basis of direct watching of hair type as well as their growth in cases who are suspected to have hisutism, the treaing physician has to decide whether hair are of terminal coarse or vellus type, besides if a male type of pattern is present as diagnosis is a subjective process. If the vellus hairs are the only ones increased, it does not point to hyperandrogenemia, and can be caused by familial or ethnic causes, abnormalities of corticosteroid, GH, or Thyroid hormone production, or can be druginduced. Though one can easily distinguish vellus hair from terminal hairs because of different texture, thicknessas well as pigmentation many patients of hyperandrogenism will have increased hair of bothtypes. The term hypertrichosis mainly refers to excessive growth strictly. Initially Ferriman and Gallwey introduced the methods of determining the degree of hirsutism visually, using scoring based on density of terminal hairs in 11 body sites which included upperlip, chin, chest, upper back,lower back,upper abdomen lower abdomen, arm, forearm, thigh and lowerleg in 161women between ages of 18-39 years attending their OPD [1].

Scoring was from 0 (absence of terminal hair to 4 extensive terminal hair growth) and they found scoring over forearm and lowerleg was found to be less sensitive, or less sensitive to androgens,thus a modified Ferriman and Gallwey method was introduced which excluded forearm and lowerleg [44,45]. Hair, growth over sideburns, lower jaw, upper neck and buttocks has been introduced in recent studies [46]. Originally Ferriman and Gallwey found the inclusion of only 9'hormonal' skin areas after excluding lower legand forearm were taken into consideration. Of the 161 women having a score above $5,4.3 \%$-of these $9 \%$ had a score of $>7$ and $1.2 \%$ had a score $>10$ [1].

Thus considering a score $>8$ is supposed to represent hirsutism.Problem was that these initial studies were mainly carried out in white people. Though the variations in the distribution, number,or androgen sensitivity of hair follicles is normal, individuals in various racial and ethnic groups needs to be defined in a better way. The information regarding prevalence of hirsutism in these various groups is very little. Hence [47] retrospectively performed a study on 369 consecutive reproductively aged black $(n=195)$ and white $(n=174)$ women who had been examined at the time of their employment by their preemployment physician [48]. They used a modification of Ferriman-Galleyway method [46], in which $<9$ body areas were assessed. Their observation was $7.6 \%, 4.6 \%$ and $1.9 \%$ demonstrated a modified Ferriman Galleyway score $>=6$, 8 or 10 respectively.

The overall s core which is used to cutoff would fall with the decrease in number of areas assessed (or the maximum score assigned to each area) is reduced.e.g. 300 unselected female medical patients using modified-Ferriman-Galleyway score where only 5 areas of body were scored like chin, upper lip, chest abdomen and thighs was studied by Lorenzo et al [45]. They did not find a score of $>5 \mathrm{in}$ any of these women.Although the exact cut off score used in white women and $7.1 \%, 6.1 \%$ and $2.1 \%$ of black women having a score $>6,8$ and 10respectively [47]. But some investigators found as compared to white women Asian women relatively rarely had hirsutism [49-51]. In view of that just absence of hirsutism cannot be considered as a feauture of absence of hyperandrogenism.

\section{Evaluation of hirsutism}

A Number of factors determine that cosmetically disturbing hirsutism will ensue

i) number of hair follicles present-since Asian women having androgen producing tumors are occasionally hirsute in lieu of the low concentration of hair follicles/unit skin area ii) The degree of resting vellus hairs conversion to terminal adult hair by androgens iii) Ratio of growth to resting phases in affected hair iv) Asynchrony of growth cycles in aggregates of hair follicles v) The thickness and degree of pigmentation of individual hairs.

The initial factor is an increase in androgen levels (usually), which produces an initial growth stimulus and then acts for sustaining continued growth. Ultimately nearly every woman with hirsutism will have increased production of T and An [51].

Anovulatory women who are not hirsute usually show laboratory evidence of increased androgen production [52,53]. 
But the commonest complaint made by women who have increased androgen production is hirsutism. Other complaints in order of frequency are acne, increased oiliness of skin, increased libido, clitoromegaly and lastly masculinization. E xtreme androgen effects lead to masculinizaion, virilization, which is not always associated with a tumor which leads to a male hair pattern, clitoromegaly, deepening of voice , increased muscle mass and a general male like habitus.

Although Ferriman-Gallwey scoring system and its modifications have been used to quantify the amount of hirsutism, they are of little clinical use. Such systems are basically meant for studies of hirsutism, but even for that purpose they have their limitations due to subjective variability [54-56].

The biggest problem for a patient is alopecia and same is troublesome for clinician as well. Many a times it is temporary with synchronous growth and loss of hair, which can be secondary to some acute stressful event.Near the end of pregnancy or postpartum telogen effluvium often occurs.As time progresses hair growth becomes asynchronous and hair thickens again within 6 months to 1year.

In a study carried out on consecutive patients with diffuse alopecia, majority had no hirsutism or menstrual dysfunction, but they had evidence of polycystic ovaries and almost $40 \%$ showed hyperandrogenemia [57]. Patients presenting with alopecia need a thorough workup as regard to hyperandrogenemia as most of them can be treated.Besides that one should rule out thyroid hormone dysfunction or any chronic illness. Still as alopecia reflects scalp 5- $\alpha$ RA, normal circulating hormone levels should not stop one from treating $[57,58]$. As a result of ageing as well hair loss occurs, in both sexes starting at age 50 [59].

Acne also reflects raised androgen activity.About 60\%of women having normal circulating levels of androgens with acne display evidence of raised 5- $\alpha \mathrm{RA}$, in the pilosebaceous unit [60]. These women benefit from antiandrogen treatment.

Acanthosis nigrans (AN) in an overweight patient, along with hirsutism-is a marker of insulin resistance and hyperinsulinemia. This is a grey brown velvety skin discoloration of the skin present usually in neck, groin and axillae; but vulva is a very common site in hirsute women. AN indicates the requirement for testing the glucose metabolism. Thus hyperinsulinemia requires serious consideration, in hyperandrogenic women.

Commonest clinical problem is the hirsute woman with irregular cycles, with the onset of hirsutism during teenage years/in ealy 20's, which worsens gradually once of longstanding duration [50]. Almost 70\%of anovulatory women develop hirsutism. The characteristic picture can usually be diagnosed by history and detailed examination.

With a good history some rare conditions can be diagnosede.g.environmental factors producing chronic irritation or reactive hyperaemia of the skin, use of drugs, changes associated with Cushing's Syndrome, acromegaly, or even the presence of pregnancy (indicating a luteoma). Hirsutism can be caused by methyl testosterone, phenytoin, diazoxide, danazol, cyclosporins, and minoxidil. Drugs which are not typically androgens cause fine hairs, which are distributed over trunk and face diffusely, also referred to as hypertrichosis. The 19-nortestosterone in the current low dosage oral contraceptives rarely (if ever) cause acne or hirsutism. DHEAS or An, which are available as food supplements, raise T levels in women and thus can be a cause for hirsutism, even at low doses used.

If a patient over 25 develops very rapid development of hirsutism which progresses to maculinization over several months to a year usually points to the presence of an androgen secreting tumor.

Late onset NCAH which is caused by enzymatic deficiency, present in adult life is rare, and gets dia gnosed only in 1-5\% of hyperandrogenic women [61,62]. The classical adrenal hyperplasia (CAH) which can lead to hirsutism usually gets diagnosed before puberty. If hirsutism occurs in childhood, usual cause is $\mathrm{CAH}$ or androgen producing tumors. Genetic causes like Y containing mosaics, or incomplete androgen sensitivity, produce signs of androgen stimulation at puberty.

If during pregnancy virilization is occurring one should suspect a luteoma, which basically is not a true tumor, but occurs only because of excessive reaction of the ovarian stromato even normal level of HCG [63]. In trophoblastic diseases one sees theca-lutein cyst salso called hyperreactio-luteinalis are always bilateral, while solid luteoma is usually unilateral, being present in $45 \%$ and always associated with pregnancy $[64,65]$. In $30 \%$ of pregnancies associated with theca-lutein cysts virilization occurs. Hyperreactio luteinalis is also seen with the high titres of HCG seen with multiple gestations. As luteoma regresses following delivery only worrisome thing is the fetus getting masculinized. Usually following pregnancies are normal but for occasional recurrence of maternal virilization [66]. Rare case reports of maternal virilization which has been occasssionally recurrent are reported with PCOS pregnancies, which should be regarded as examples of hyperreactio luteinalis $[67,68]$.

The presence of androgen secreting tumors is very rarely encountered along with pregnancy, possibly as raised androgens suppress anovulation [25,26]. Ultrasonography (USG) of pelvis can help in diagnosis in women suffering from virilization during pregnancy.In a solid unilateral ovarian tumor, malignancy is commonly seen.

Thus mostly it is anovulation which is usually cause of hirsutism, but a minimal tests towards ruling out ovarian tumors as well as adrenal tumors is needed.

\section{Workup for Hirsutism}

Medical History in detail and thorough physical examination are very important. Presentation of patient usually corresponds 
with ano vulation and polycystic ovaries; in the time course. A search for galactorrhea thoroughly is important in an anovulatory patient, besides testing the serum prolactin and thyroid function tests. Following that laboratory investigations are directed towards making a diagnosis of insulin resistance, adrenal hyperplasia or an androgen secreting tumor.

One starts with testing serum testosterone (T) and $17 \alpha$-hydroxyprogesterone (17-OHP). Now the testing of DHEAS is no longer considered essential.It is important to screen for TSH even in patients with alopecia. Those patients, who have severe hyerandrogenemia, may present with amenorrhea, secondary to endometrial suppression (along with a decidual response) and thus may not get a withdrawl bleed with a progesterone (P).

One of the commonest referral is regarding Cushing's syndrome, which although can cause hirsutism and masculinization it usually does not turn out to be the diagnosis in most of the cases. Only if suspicion high a screen for Cushing's syndrome is indicated.

\section{Evaluation of cushing's syndrome (CS)}

Persistent oversecretion of cortisol is known as CS.Their are five ways by which it can be produced

a. Pituirtary adrenocorticptropic hormone (ACTH) overproduction is the true CS

b. Ectopic ACTH overproduction by tumours

c. Autonomous cortisol secretion by the adrenal

d. Very rarely autonomous cortisol secretion by the ovarian tumor

e. Very rarely secretion of CRH by a tumor.

One must first make a diagnosis of CS before looking for etiology.Most important is the basal state measurement for detecting CS and 24hrs urinary free cortisolexcretion (10-90 $\mu \mathrm{g}$ ) and late evening plasma cortisol level $<15 \mu \mathrm{g} / \mathrm{dL}$. ls Start with a single dose overnight dexamethasone test where dexamethasone $1 \mathrm{mg}$ is given orally at $11 \mathrm{pmand}$ a plasma cortisol is drawn at $8.00 \mathrm{am}$ in the nexr morning. A value $<$ than $5 \mu \mathrm{g} / \mathrm{dL}$ rules out CS.CS is unlikely, with intermittent values of $5-10 \mu \mathrm{g} / \mathrm{dL}$, whereas having a value $>10 \mu / \mathrm{dL}$ Is diagnostic of adrenal hyperfunction. The patients who show normal suppression in the single dose overnight dexamethasone of CS is negligible<1\% [69]. False positive rate is $13 \%$ in obese patients.If single dose overnight test is abnormal, establish the diagnosis by measuring $24 \mathrm{hr}$ urinary free cortisol.

Abnormal single dose overnight suppression can make the diagnosis by getting $24 \mathrm{hr}$ urinary free cortisol. With the 2 day low dose suppression test final diagnosis gets confirmed.Giving dexamethasone $0.5 \mathrm{mg}$ every $6 \mathrm{hrs}$ x 2 days oncebaseline $24 \mathrm{hr}$ urinary 17 hydroxy steroid as well as free cortisol gets measured. Patients with CS will not get the urinary steroids $<25 \mathrm{~g} /$ day and free cortisol<10 $\mu$ g on 2ndday of dexamethasone suppression.
Once one combines the lower dose test with the 24hr urinary free cortisol, diagnosis of CS gets confirmed.Having a $24 \mathrm{hr}$ urinary free cortisolof $250 \mu \mathrm{g} /$ day is almost diagnostic, while a level of $200 \mu \mathrm{g}$ gives $90 \%$ accuracy.

There is pseudo cortisolism in patients having mild hypercortisolism, secondary to alcoholism, anorexia nervosa, bulimia, severe obesity, extremes of stress, depression. Mostly not necessary if one combines the low dose dexamethasone suppression with CRH suppression, one can accurately distinguish the true CS from the pseudo hypercortisolism due to these various conditions [70]. 2days following low dose dexamethasone suppression, a single plasma cortisol level is obtained after $15^{\prime}$ of giving $1 \mu \mathrm{g} / \mathrm{Kg}$ iv.A cortisol level $>1.4 \mu \mathrm{g} / \mathrm{dl}$ needs further evaluation.

One can find out the etiology of CS after combining a high dose dexamethasone suppression (2mg every 6hrs)x2days ,and urinary 17hydroxy steroids and cortisol levels on the 2nd day comparing it with the basal levels.Once this basal ACTH concentration can be measured in the blood $>20 \mathrm{pg} / \mathrm{ml}$, an ectopic ACTH producing tumor is not likely, if the urinary steroids decrease by at least $40 \%$. Cushing's disease is present if blood ACTH level s of plasma are $>50 \mathrm{pg} / \mathrm{ml}$, which suggests an ectopic ACTH release, while a low level $<5 \mathrm{pg} / \mathrm{ml}$ suggests an autonomous cortisol secretion.

To diagnose adrenal tumors imaging is very reliable and accurate.Besides that it predicts which patient maybe having ectopic ACTH producing tumor, by finding bilateral enlarged adrenal glands in such patients.Computed tomography(CT) scanning of adrenal gland gives better resolution ,being preferred over magnetic resonance imaging(MRI) and ultrasonography(USG).

Evaluating CS can give inconclusive results and a failure to recognize occult, ectopic ACTH secreting tumor can lead to unnecessary pituitary or adrenal surgery.Bilateral venous sampling obtained from the inferior petrosal sinuses (samples draining the blood draining from the pituitary gland)for the measurement of ACTH ,before as well as following CRH stimulation is a good method of achieving accurate diagnosis of a pituitaty origin of the ACTH [69]. Roughly 15\%of patients with ACTH dependent CS willhave anoccult, ectopic source for ACTH. Mostly these ACTH secreting lesions are present in thorax (usually small cell lung carcinoma) and some are in abdomen. One recommends petrosal sinus sampling in all patients with ACTH dependent CS, who do not have an obvious adenal tumoron imaging. Very rare cause of CS is the autonomous production of cortisol by an ovarian tumor [71]. It is recommended to do chest and abdomen imaging in for all atypical presenting cases

\section{DHAS}

DHAS is almost exclusively derived from the adrenal gland and circulates in higher concentrations as compared to any other steroids. Thus it gives a direct measure of steroid androgen 
activity which correlated with the urinary 17-keto steroids clinically. It has an upper limit of $350 \mu \mathrm{g} / \mathrm{dL}$ in most laboratories, but because of laboratory to laboratory variations one should consider the normal reference range of the laboratory.

Just a single DHAS sample is enough for hirsuism evaluation, which needs no correlation for body weight, creatinine excretion or episodic variation. Variations go to a minimum as this has a high circulating concentration and has a long half life. A slow turnover ensures stable pool in hirutism. Hence DHAS serves as a prehormone in hair follicles, providing substrate for the hair follicle synthesis of androgens [72]. Both 17KS and DHAS levels are raised in association with hyperprolactinemia [73,74]. Once prolactin is suppressed with the use of dopamine agonists these levels return to normal.Also besides raised DHAS levels $\mathrm{T}$ is found in hyperprolactinemia $[75,76]$. Hence it is very important to look for galactorrhea in all anovulatory patients. Androgen levels are secondary to persistent anovulatory changes, caused by hyperprolactinemia, although direct effects of prolactin on the adrenal, ovary or SHBG are possible.

With normal DHAS levels, adrenal tumors are rare. Further evaluations of such cases are dependent on having markedly raised $\mathrm{T}$ [77]. These tumors, which are rare, respond to $\mathrm{LH}$, which suggests they maybe derived from embryonic rest cells.

One frequently finds moderately increased DHAS levels in anovulatory women with PCOS. If 17 -hydroxy progesterone is normal, it is not worthwhile to search for an adrenal enzyme defect. Mostly clinical experience tells that moderate increases in DHAS are associated with anovulation and suppression of ovarian function restores the DHAS levels to normal.

DHAS levels $>700 \mu \mathrm{g} / \mathrm{dl}$ has been accepted as abnormal adrenal function.But these levels are rarely found. Even if these DHAS $>=700 \mu \mathrm{g} / \mathrm{dl}$ is confronted; it will be associated with an increased $\mathrm{T}$, either by direct secretion or peripheral conversion from DHAS. Hence in absence of CS, measurement of DHT is sufficient to screen for adrenal abnormalities.Getting an imaging study of adrenal glands once $\mathrm{T}$ is raised, it is more cost effective than measuring DHAS levels in all hirsute women.

\section{Non classical Adrenal Hypeplasia}

CAH occurs secondary to an enzyme defect leading to raised androgen production. It is a condition having prenatal onset, is very severe and gets inherited as an autosomal recessive disease. When it occurs later in life, the disease has a milder form, when it is known as late onset, partial or NCAH [78-80]. The symptoform cryptic adrenal hyperplasia gets discovered only by biochemical screening.

Although all the enzymes recruited for conversion of cholesterol to cortisol can be affected, commonest ones are 21-hydroxylase ( $p$ 450c21), $11 \beta$-hydroxylase (p450c 11) and $3-\beta$ hydroxysteroid dehyrogenase.

\section{1-hydroxylase defect}

These Patients presenting with late onset adrenal hyperplasia due to a

21-hydroxylase deficiency, respond to ACTH stimulation in a modest fashion, between the classical adrenal hyperplasiahomozygotes response and the mild heterozygote response.

A 21-Hydroxylase deficiency is the commonest autosomal recessive disorder, which surpasses, cystic fibrosis as well as sickle cell anemia. Variable clinical presentations can be seen and it may appear and disappear over time. Thereforethe diagnosis needs laboratory evaluation.

Genetic diagnosis for known mutations like Cyp21 gene is not discussed here. The 3 reasons that why it should be diagnosed arei) Therapy which is accurate should be used, as it maybe longterm.

a. Pregnant women with these conditions need genetic counseling, along with assessment of asymptomatic offspring.But for that the father's carrier status is important for estimating the risk to the fetus. Though the risk for having a child with $\mathrm{CAH}$ is very low, the couple must get paternal testing for homo/hetrozygosity.If father is positive, prenatal diagnosis and treatment is reasonably required.

b. These Patients might be having a cortisol deficiency, theoretically when they experience severe stress, however this has not been a clinical problem.

\section{Other enzymatic defects}

$3-\beta$ hydroxyl steroid dehydrogenase (type1) deficiency can present in both ovary and adrenal. Thus significant androgen production is not seen, but enzymatic activity remains intact in peripheral tissues.Hence hirsutism, present in this deficiency is due to a target tissue conversion of the increased secretion of precursors [81]. Unlike 21-hydroxylase deficiency, no genetic markers are currently available. Thus for diagnosis, an ACTH stimulation test and demonstration of altered conversion of 17-hyroxy pregnenolone to 17 hydroxy $\mathrm{P}$ (170HP) ratio is required. Though increased $170 \mathrm{HPresponse}$ to $\mathrm{ACTH}$ stimulation is common in women with hyperandrogenism, response is consistent with adrenal hyperactivity and not an enzymatic deficiency $[82,83]$. Molecular studies fail to find mutations in the genes for the $3 \beta$-hydroxy steroid deyrogenase in patients appearing to have mild-moderate deficiency in $3 \beta$-hydroxy steroid dehydrogenase [84-86]. The deficiency present is so subtle, that accurate diagnosis is not essential. Usual therapeutic approach to hirsutism will be effective.

$11 \beta$-hydroxylase deficiency is very rare and gets diagnosed at a younger age.It is not worthwhile to measure the 11 deoxy cortisol response to ACTH stimulation in adult hirsute women, which is just an effort to detect a very rare deficiency [87]. 


\section{7 hydroxy Progeterone (170HP)}

1-5\% of women presenting with hirsutism show a biochemical response which is consistent with the less severe form of the adrenal hyperplasia namely 21-Hydroxylase variety[64,65,88-90].Thus the relative frequency of NCAH requires the routine screening of $170 \mathrm{HP}$ screening of women who complain of hirsutism.Routine use of ACTH stimulation test is not warranted [64,91]. CYP I f CYP21 mutations ,are heterozygous it does not increase the risk, of clinically significant hirsutism[92]. To make 170HP testing cost effective, a decision of ACTH stimulation test can be based on clinically significant hirsutism [87].

If there is a strong family history of androgen excess, it suggests the presence of an inherited disorder. Hirsutism due to an adrenal enzyme defect is more severe, beginning at a young age, mainly at puberty.Short stature and very high levels of androgens signify a more severe problem. Finally it is worth considering that with normal baseline steroid levels, even if a woman has subtle enzyme defects, the management of the problem does not require its discovery.

170 HP needs to be measured early in the morning, to avoid later deviation due to the diurnal pattern of ACTH secretion.The baseline 170HP levels should be <200ng/dl [64,93,94].>200ng/ $\mathrm{dL}$ and $<800 \mathrm{ng} /$ dlneeds ACTH testing.Levels $>800 \mathrm{ng} / \mathrm{dL}$ are virtually diagnostic of 21 hysroxylase deficiency.DHAS levels are usually normal.Hallmark of NCAH are increased levels of 170HP, with a dramatic increase after ACTH stimulation[89]Still an increased baseline levels of $170 \mathrm{HP}$ are often not impressive(eg overlapping)with those found in women with PCOS due to anovulation, and a simple ACTH stimulation must be utilized.

\section{ACTH stimulation test}

Intravenous synthetic ACTH(Cortrosyn)is given in a dose of $250 \mu \mathrm{g} .170 \mathrm{HP}$ is measured at $0 \mathrm{hrs}$ and after $1 \mathrm{hr}$ at $8 \mathrm{AM}$ at any time of the menstrual cycle.The $1 \mathrm{hr}$ value should be plotted to predict the genotype ,whether homozygote or heterozygote forms of the 21hydroxylase deficiency[95].Dexamethasone preparation at night before is unnecessary [96]. 21 hydroxylase deficiency in case of heterozygote carriers is reflected by ACTH stimulated levels of $170 \mathrm{HP}$ upto $1000 \mathrm{ng} / \mathrm{dl}$,patient having late onset deficiency have levels $>1200 \mathrm{ng} / \mathrm{dl}$.

For diagnosing $3 \beta$ hydroxy steroid deficiency in the ACTH stimulation test, one measures $170 \mathrm{HP}$ and 17hydroxy pregnenolone ratio. An abnormal 17 hydroxy pregnenolone/170HP ratio is usually $>6$ [80]. In this deficiency there is markedly raised DHAS, although serum $\mathrm{T}$ is just normalor mildly increased. To diagnose $11 \beta$ hydroxylase deficiency deoxy cortisol will be increased, which is normal in 21 hydroxylase defects.

\section{Adrenal gland and anovulation}

Adrenal suppression has been known to induce both ovulation as well as regular menses, because of which empiric therapy had been advocated in the past. Late onset adrenal hyperplasia cannot explain all patients presenting with raised DHAS. It is important to question, is adrenal gland the primary culprit or is raised DHAS secondary to the altered hormonal milieu associated with anovulation. There is a possibility that increased adrenal gland activity is due to effect of estrogen on $3 \beta$ hydroxy steroid dehydrogenase leading to anovulation.Although a lot of work has been done to prove the role of $E$ on $3 \beta$ hydroxy steroidhydrogenase to prove adrenal insufficieny.Despite these works to d prove the role of $\mathrm{E}$, there are studies both for [97101], and against [102-106] this hypothesis.

This has been compared to its similarity of decreased levels of $3 \beta$ hydroxy steroid dehydrogenase in fetal gland and raised DHAS secretion by the fetal adrenal cortex secondary to E $[107,108]$. But studies against this prove that ACTH levels are not raised in adult anovulatory women $[109,110]$, although the period of raised ACTH responses would exist only till normal cortisol levels are reachieved, which is the new set point. Studies of anovulatory female, with inceases in levels of DHAS, indicate that the raised adrenal activity is because of a mechanism, within the adrenal gland. It is not secondary to raised pituitary response to raised $\mathrm{CRH}$ and are not because of increased adrenal response toACTH stimulation [111].

Both androgenand estrogens inhibit $3 \beta$ hydroxy steroid dehydrogenase in concentration expected to be in adrenal gland although it is difficult to achieve with exogenous administration. Stimulation [112].Therefore adrenal secretion changes may show the varying actions of steroids especially $\mathrm{E}$, in different layers of the adrenal cortex [113].Steroidogenesis not getting affected by giving exogenous androgens supports this hypothesis [114].

Therefore this hypothesis that raised adrenal activity in anovulatory women is induced and maintained by a steady state of E state, which is seen in anovulation. In patients of PCOS associated with increased adrenal androgen activity, there exists a correlation between adrenal sensitivity to ACTH levels and E levels [115]. But this may be secondary to another mechanism rather than affecting the $3 \beta$ hydroxy steroid dehydrogenase. Ovarian suppression with GnRH agonists has been used with the hope to clarify this puzzle by assessing function of adrenal after suppression of ovarian steroid production. Short term suppression for 3-6mths does not have any impact on the adrenal androgen production [116,117]. But these studies did not include women with raised DHAS levels. Once women who had raised DHAS level received 3mths of GnRH agonists, in some but not allwomen, DHAS levels were suppressed $[83,116]$.Their has been a suggestion that raised adrenal androgen production has been due to $\mathrm{p} 450 \mathrm{c} 11,17,20$ lyase hyperactivity and in others it is a response which is acquired secondary to a steady state of $\mathrm{E}$ in anovulatory women [83,118]. 92 consecutive women were studied with hirsutism who on ACTH stimulation showed that the steroidogenic response which is acquired secondary to anovulatory hormonal steady state was inconsistent with that of an inherent disorder of p450c17 [119]. In these women with 
p450c 17 increased activity, underlying the disorder maybe secondary to insulin resistance which leads to hyperinsulinemia [120]. Both insulin and IGF1 receptors are present on adrenal cells. On infusion of insulin in women it $=>$ a decrease in adrenal 17, 20 lyase ( $\mathrm{p} 450 \mathrm{c} 17)$ activity which suggests that insulin reduces the production of adrenal androgen $[121,122]$.

Simple inverse relationahip between insulin and adrenal androgen levels in anovulatory women does not exist [123]. In a study by Landay et al. [124] data was collected regarding androgen excess between 1987-2002, where 749 patients were diagnosed to have PCOS by NICHD criteria, and it was found that insulin had a direct effect on the severity of hirsutism in PCOS and its effects appeared to be synergistic with TT. Over $90 \%$ of the variations in $\mathrm{mFGscore}$ was not related to the factors studied and reflect probably the intrinsic factors related to pilosebaceous unit function or sensitivity to other factors which have not been assessed till now [124] Studying the effect of bilateral oophorectomy on adrenocortical function in women with PCOS Azziz et al. [125] 2013 found that total T, free T, and estrone levels decreased, while their was a significant raise in FSH levels following oophorectomy.There were no significant differences found in E2, DHEAS, or SHBG. Basal as well as stimulated androstenedione $(\boldsymbol{\Delta} 4 \mathrm{An})$ was raised to a significant extent. No significant changes were found in DHA, DHEAS 60 or cortisol (F0); F60 and $\mathbf{\Delta}$ F levels showed a tendency to increase following oophorectomy. Although levels were not significant. Thus they concluded that ovarian factors do not seem to contribute significantly to the adrenocortical dysfunction of PCOS [125]. Age related decrease in DHAS may be related to insulin resistance [126].

In Anovulatory women ovulation induction can be explained in part by an ovarian androgen production by circulating DHAS. A considerable portion of circulating $\mathrm{T} p$ roduction by the ovarian follicles can be attributed to circulating DHAS which serves as a substrate of prehormone [127] Hence dexamethasoe suppression cannot separate adrenal and ovarian T secretion, in that both the glands are involved in a com-plex interacton, with DHAS providing atleast one mechanism for this interaction.

Because of inconclusive studies once a $170 \mathrm{HP}$ of $200 \mathrm{ng} / \mathrm{dLis}$ attained, there is no point of considering possibility of primary adrenal enzyme problems below that and there is little use of measuring DHAS. One can treat mild adrenal enzyme defects by usual methods and do not require glucocorticoid administration.

\section{Bidiopathic Hirsutism(IH)}

The deinition of IH has varied in the past three decade. Diagnosis of IH T be used only in hirsute patients with normal ovulatory function and circulating androgen levels.Just having a history of regular cycle is not enough to exclude ovulatory dysfunction. Upto $40 \%$ of such hirsute women are anovulatory. Strictly if criteria areused the diagnosis of IH will be left to $<20 \%$ of hirsute women. Studies of Asch et al of the 132 (100\%), hirsute women 64 (48\%) had regular cycles, while rest 68 (52\%) did not have regular cycles.39 (61\%) had normal ovulation as seen by BBT or luteal phase P, while $25(39 \%)$ did not have normal ovulation. Of the patients having normal ovulation 22 (56\%) had normal total and free T and DHAS, while 17 (44\%) did not have normal levels.These 22(56\%) patients were the ones having IH,while the other 44\%having normal levels and 39\% not having normal BBT,were considered as functional androgen excess[128].

The pathophysiology of IH Is thought to be due to an increase in skin 5- $\alpha$ RA activity, probably in both enzymes type I and type II and possibly a defect in androgen receptor function. These patients respond to antiandrogens which inhibit 5- $\alpha$ RA activity. New therapies like new biological modifiers may play an important role in giving more effec tive therapy for unwanted hair. More investigations are needed into genetic, molecular and metabolic aspects of the disorder reviewed by Azziz et al [129].

\section{Treatment of hirsutism}

Treatment is addressed in interrupting the steady state of excess production in view of practically all patients presenting with a steady state of anovulation and thus hyperandrogenemia. The production of androgens in hirsute women usually is a LH-dependent process. Ovarian steroidogenesis suppression depends on an adequate suppression of LH. Besides the inhibitory actions of the progesterone component (P), a combination estrogen-progestin contraceptive gives a further benefit by causing an increase in SHBG, due to the estrogen component. Araised SHBG causes increased binding capacity of androgens, which is associated with decrease in circulating free T levels. $\mathrm{P}$ in estrogen-progestin OC's also inhibits 5- $\alpha$ RA, in the skin, which further contributes to the clinical impact of OC's in hirsutism[130]. Even Estrogen-progestin Contraception can be applied transvaginally or transdermally and be effective.

Use of low dose OC's are effective in treating both acne and hirsutism [131,132]. Even the same beneficial effects with preparationshavinglow doselevonorgestrel, which waspreviously recognized to cause acne at high doses can be used $[132,133]$. Preparations having desogestrel,gestodene,norgestimate, are associated with a >SHBG,which further causes marked fall in circulating $\mathrm{T}$ levels, but studies comparing these preparations find no difference in various androgens measured amongst these $[131,134]$. Although in theory one presumes that these preparations should be more effective clinically in treating acneor hirsutism, in clinical studies no difference in response was found [135]. Thus all low dose preparations, with the combined effect of an increase in SHBG and a decrease in free $\mathrm{T}$ production are practically similar in response once used for a year or more. Using a combination of ethinyl E2in combination with drosperinone may have greater antiandrogenic effects and lesser metabolic side effects as compared to other OC's having other P combinations. This is in view of structural similarily of drosdperinone with spironolactone.However one has to use 
them judiciously where hyperkalaemia is susapected or drugs which interact, and side effects like hepatic dysfunction like other OC's should make a judicious use 136reviewed by Mathur et al. [136].

Even in women who are placed on antiandrogens, OC's give a control of cycle along with contraception. If OC'are contraindicated or patient is reluctant to use them medroxy progesterone acetate (MPA), gives good results in a dose of either $150 \mathrm{mg} / \mathrm{month}$ and respond poorly to or daily oral MPA in dose of $10-20 \mathrm{mg} /$ day. Mechanism of action of MPA is that it suppresses gonadotrophins, less intensely and thus the ovarian follicular activity continues. LH suppression is significant, however T production gets decreased. Although to a leser degree than OC's. Besides the $T$ clearance from circulation gets increased [137]. This is secondary to an induction of liver enzymes activity although MPA suppresses SHBG, so that less $\mathrm{T}$ is bound, but because total $\mathrm{T}$ production is decreased to such great extent that actual amount of free $\mathrm{T}$ is decreased [138].Hence comparable overall results is achieved with OC and MPA.

Since hair follicle cycle change takes time, response to treatment is slow. One must warn the patient that hormonal suppression of at atleast $6 \mathrm{mths}$ is essential before a noticeable decrease in hair growth occurs. Hence combined treatment with electrolysis or laser hair removal is not recommended, till hormonal suppression has been used atleast for 6months. Although new hair follicles will no longer be stimulated to grow, hair growth which has been established does not disappear, with hormone treatment alone.

Temporarily one can achieve by shaving, tweezing, waxing or depilatories [139].These methods do not stimulate or increase rate of hair growth as has been the usual belief in the past. But they need to be used repeatedly as they do not effect inherent hair growth. For permanent hair removal electrocoagulation of the dermal papilla is required. One needs to emphasize that electrologists use disposable needles.One can also use laser for hair removal [140]. Women with dark hair and light skin, get the best results, because absorption of light by dark pigments produce local thermal damage to the hair follicle.

Effects of treatment may not be apparent till previously established hairs are removed. Hence combining ovarian suppression, preventing new hair growth and electrolysis/laser hair removal yields most complete treatment. Only in resistant patients other methods need to be employed, despite 1 year of treatment.Mostly DHAS levels get suppressed by progestational treatment $[132,133]$. Although the exact mechanism of action is not clear. If original cause for increased DH AS secretion is a steady state of oestrogen (E) causing anovulation, then change in endocrine, milieu of adrenal gland brought about with ovarian steroidogenesis restores normal adrenal secretory pattern. E-P OC may also produce subtle but significant alterations in ACTH secretion or response of the adrenal gland.
Suppressing adrenal gland to produce ovulation in some cases having anovulatory cycles is attributable to lowered circulating androgen levels due to a decrease in contribution of adrenal glands along with a decrease in amount of DHAS available for conversion to $\mathrm{T}$ within the ovarian follicle. Thus within the follicle androgen levels are decreased and hence prevents inhibitory actions of androgens on follicular growth and development. But this is not the replacement for the first line of drug for inducing ovulation, namely clomiphene. As far as hirsutism treatment is concerned $\mathrm{P}$ suppression of ovarian steroidogenesis remains the first treatment of choice. Suppression of adrenal needs to be reserved only for patients having an established diagnosis of adrenal enzyme deficiency.

In older women where OC's are contraindicated for some reason only then a surgical option is considered.Persistent hirsutism growing in severity is an indication for TAH with BSO. Similarly patients with hyperthecosis are usually older and respond poorly to ovarian suppression.

Association of hyperinsulinism with hyperandrogenemia needs correction of hyperinsulinemia and obesity leading to insulin resistance besides metformin to control the same. In a multi centre study Roth et al studied the effects of ovulation induction with clomiphene, metformin or both in 626 women with PCOS and infertility and did not find any change in hirsutism with these ovulation induction techniques as monitored by the mFG criteria [141Roth].

\section{Other methods of treatment}

Spironolactone:This is a diuretic which inhibits aldosterone. Regarding its actions in hirsutism i)it can directly inhibit 5- $\alpha$ RAii) Inhibition of steroidogenesis can be obtained by an effect on cytochrome p-450 system, but the suppressive effec ts on steroids are so variable that the receptor blocking effect is one of the most important actions [142]. Because of this reason, cortisol, DHA and DHAS are not changed although androstendione levels are decreased [143]. Effect on hirsutism is dose related, with a better effect with $200 \mathrm{mg} /$ day [144-146]. After some time it can be gradually reduced to a maintainance dose of $25-50 \mathrm{mg} /$ day. Its effects are also slow, just like progestational agents, and only one can see their effect after 6 months of treatment. Side effects are minimal, which are diuresis in the initial few days, with occasional fatigue, and dysfunctional uterine bleeding. Because of this a progestational agent needs to be added for anovulatory state to avoid abnormal uterine bleeding, as well as prevent excessive endometrial hyperplasia because of possibility of hyperkalaemia, one should not use it in a diabetic patienr, obese or in patients on drugs which increase potassium levels. It is usually used if patients find OC's unacceptable/ disappointing response. One can combine the peripheral action of spironolactone with OC's to get a result, more dramatic, but clinically combined treatment has not shown more efficacy than single agent therapy $[147,148]$. Acne can be treated by a cream 
having 2-5\% spironolactone [149]. No systemic side effects are seen with this.

Since once androgens are suppressed with spironolactone there are chances that anovulation gets corrected and patient may get pregnant due to the ovulation spontaneously and fear of feminization of a male fetus, but clinically such effects have not been seen [150].Still better to keep patient on contraception once on spironolactone therapy.

Flutamide: Flutamide (Eulexin) acts at the receptor level, being a nonsteroidal antiandrogen [151].It inhibits hair growth directly without many sideeffets, commonest being dry skin. Still hepatotoxicity is a possibility [152]. Because of this a low dose approach is recommended as although rare it is a severe side effect. Daily dose of $250 \mathrm{mg}$ can have remarkable effects on hirsutism within 6months [153]. LFT should be monitored despite low c hance of liver sideeffects. Comparing $250 \mathrm{mg}$ bd of flutamide and spironolactone 100mg OD did not show much difference [154]. One needs to combine OC's which is due to antiandrogen effect having the probability of effecting male fetus development.Although due to hepatotoxicity it is not the drug of choice but for alopecia ,in a study of finasteride, cyproterone, only $250 \mathrm{mg}$ fluta mide was found to be effec tive [155].

Finasteride: This inhibits $5 \alpha$ RA-thus blocking T to DHT conversion. This enzyme exists in two forms, type 1 and type 2 , each getting encoded by different genes. Type I is found in skin and typeII reductase is predominantly present in reproductive tissues [156]. Proscar the pharmaceutical preparation used for prostate cancer inhibits both enzymes, although its potency for hirsutism treatment as well as alopecia is limited as it is less effective for type I enzyme. Dose of $5 \mathrm{mg}$ inhibits 4 hirsutism [157], without side effects although a smaller dose available as Propecia (1mg) has been available for men with alopecia. In a RCT all three finasteride, flutamide and spironolactone $100 \mathrm{mg}$ were equally ef fective $[158,159]$. But in another RCT spironolactone $100 \mathrm{mg} /$ day had greater efficacy as compared to finasteride [160]. Though effective finasteride was <effective than flutamide $250 \mathrm{mg}$ and spironolactone [161]. Both forms of finasteride have not been found to be of use in post menopausal women with alopecia [152,162]. Biggest advantage of finasteride is lack of side effects butsince DHT inhibition can effect development of urogenital si nus and urogenital tubercle into male external genitalia, urethra and prostate, it is essential to use contraception while using it for hirsutism. Tehvilian found that finasteride gel $0.25 \%$ had good absorption as well as solubilityand hence can be used efficiently without side effects encountered with the oral drug.Thus it can be used in patients with IH [163].

Cyproterone Acetate (CA): CA is a potent progestational agent as its use inhibits both gonadotropin secretion as well as block androgen binding to its receptor. It has been used in an E-P contraceptive, known as Diane $(2 \mathrm{mg}$ CA and $50 \mu \mathrm{g}$ ethinyl estradiol (E2))or Dianette35 containing $2 \mathrm{mg}$ CA and $35 \mu \mathrm{g}$ E2. In reversed sequential regimen $\mathrm{CA}$ is given as $50-100 \mathrm{ng} / \mathrm{dL}$ from d5-14 with E2 from d 5-25[164].A comparison of Diane with higher doses of CA although their effect was greater but was not of clinical significance.Side effects were similar [165]. Effect of $2 \mathrm{mg}$ dose of CA in Dianette was equal to that of high dose [166]. Side effects are fatigue, oedema, loss of libido, weight gain and mastalgia.Facial hirsutism improvessignificantly in 3d month of treatment.Amonophasic low dose OC combined with $100 \mathrm{mg}$ apironolactonewas as effective as the reverse sequential regimen of 50-100 $\mu \mathrm{g}$ of CA and E2 [167-170].

\section{Conclusion}

Hirsutism, defined as terminal hair on the body of a female, aff ects $5-10 \%$ of women. Of these the commonest cause is anovulation secondary to PCOS, which is a fairly common disorder affecting $6-10 \%$ of women of reproductive age group. Although ovary is the main source of androgen excess, evidenc e with synthesis of androgens from adrenals is exaggerated with $25-50 \%$ of PCOS patients showing an adrenal androgen exces. Androgens can stimulate hair follicles, producing conversion of vellus type of hairs to terminal hairs. The degree of production of of dihydro progesterone (DHT) is determined by the degree of $5-\alpha$ reductaseM (5- $\alpha-\mathrm{RA})$ and consequently the effect.

The degree of production of of DHT is determined by the activity of $5-\alpha$ RA.Their are two kinds of $5 \alpha$-RA isozymes. The exact degree of activity on facial hair and abdominal skin in hirsutism is unclear. Besides PCOS other hormonal causes like thyroid hormone deficiency, growth hormone deficiency, or hyperprolactinemiar, hyperinsulinemia which may act on their own or synergistic increase with adrenal androgens. Aserum $\mathrm{T}$ over $2 \mathrm{ng} / \mathrm{ml}$, warrants exploring for an androgen secreting tumor. Although in some cases oft resort PCOS massive virization deepening of voice, receding hairline with a normal karyotype has been reported in the absence of a tumor, which just needs simple treatment efforts of controlling hyperandrogenemia. Idiopathic hirsutism is diagnosed as a last resort even in women having normal cyclesand is not defined as familial hirsutism. Treatment recommended initially is an oral contraceptive with low dose ethinyl estradiol $(30 \mu \mathrm{g})$, preferably with drosperinone or other progestogens. Treatment is slow and minimum $6 \mathrm{mths}$ of treatment is needed to observe any demonstrable change.Any treatment like laser or electrolysis should be postponed till this period.Preferrably one uses upto one year of this treatment. If no response with OC's add antiandrogens like spironolactone or finasteride, although spironolactone is preferred. One can choose drug which has minimum side effects and is cost effective. GnRH agonists remain the drugs as a last resort. Similarly cyproterone regimens may be used, Topical applications of spironolactone, fiasteride may be of help if systemic side effects troublesome. Eflornithine cream for 8 weeks has been advocated but the authors have not found them to be very effective alone. Drugs like cimetidine or ketoconazole have an effect but are not drugs of choice. 


\section{References}

1. Hemalatha AL, Konanahalli $P$ (2005) Bilateral malignant Brenner tumor of ovary. J Obstet Gynecol India 55(1): 81-82.

2. Katke RD, Kiran U, Saraogi M, Sarode S, Thawal R (2014) Giant borderline mucinous cystadenoma with previous 3 caesareans. J Postgraduate Gynaecol Obstet 1: 1-4.

3. Green GE, Mortele KJ, Glickman JN, Benson CB (2006) Brenner tumors of the ovary sonographic and computed tomographic imaging features. J Ultrasound Med 25(10): 1245-1251.

4. Arey LB (1961) The origin and form of the Brenner tumor. Am J Obstet Gynecol 81: 743-751.

5. Katke RD (2015) Huge mucinous cystadenoma of ovary with massive third degree uterovaginal prolapse in postmenopausal woman: rare case report and review of literature. Int J Reprod Contracept Obstet Gynecol 4(1): 255-258.

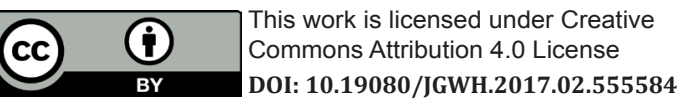

Your next submission with Juniper Publishers will reach you the below assets

- Quality Editorial service

- Swift Peer Review

- Reprints availability

- E-prints Service

- Manuscript Podcast for convenient understanding

- Global attainment for your research

- Manuscript accessibility in different formats

( Pdf, E-pub, Full Text, Audio)

- Unceasing customer service

Track the below URL for one-step submission

https://juniperpublishers.com/online-submission.php 